\title{
Inelastic Magnetic Scattering of Circularly Polarized X-rays
}

S W Lovesey

April 1996 
Enquiries about copyright, reproduction and requests for additional copies of this report should be addressed to:

The Central Laboratory of the Research Councils

Library and Information Services

Rutherford Appleton Laboratory

Chilton

Didcot

Oxfordshire

OXI1 OQX

Tel: 01235445384 Fax: 01235446403

E-mail library@rl.ac.uk

ISSN 1358-6254

Neither the Council nor the Laboratory accept any responsibility for loss or damage arising from the use of information contained in any of their reports or in any communication about their lests or investigations. 


\title{
Inelastic Magnetic Scattering of Circularly Polarized X-rays
}

\author{
Stephen W. Lovesey \\ ISIS Facility, Rutherford Appleton \\ Laboratory, Oxfordshire OX11 0QX, U.K.
}

\begin{abstract}
Circular polarization in a X-ray beam incident on a magnetic material induces a signal in the scattered beam from an overlap between charge and spin and current (linear momentum) densities. The overlap cross-section is the basis of most recent experiments with $\mathrm{X}$-ray beams performed to measure the Compton profile of unpaired electrons. Using the language of correlation functions, standard in the interpretation of neutron scattering experiments, we analyse the structure and content of the overlap cross-section, including its behaviour in the Compton limit of scattering. It is shown that, invariance of the Hamiltonian describing the electrons through a time-reversal operation and simultaneous reversal of the magnetic field leads to a very valuable statement about the field and space-parity property of the cross-section which has implications for the execution of an experiment and interpretation of empirical data.
\end{abstract}


Experimental techniques using X-ray beams, generated by synchrotron sources, are rapidly becoming very important methods for investigating the magnetic properties of materials on an atomic scale of detail [1,2]. When compared to well-established methods using neutron beams X-ray techniques provide superior quality data in some cases, and there are a few cases where there is no alternative technique. One example is spectroscopic data derived by scattering a beam of hard X-rays, with an energy larger than all atomic resonance energies of the constituents of the magnetic sample under investigation. Under appropriate experimental conditions Compton scattering is realized; the intensity of Compton scattered $\mathrm{X}$-rays is centred at an energy transfer equal to the recoil energy of an electron $\varepsilon=(\hbar k)^{2} / 2 \mathrm{~m}$ where $m$ is the mass of an electron and $k$ is the magnitude of the change on scattering in the wave vector of the X-rays. The distribution of intensity about the recoil energy, often called the Compton profile, is directly related, via a Radon transform, to the momentum density of the electrons participating in the inelastic and incoherent scattering process. Today, large sets of very high quality data are obtained, and confronted with predictions from state-of-the-art theories of electrons in magnetic materials $[3,4]$. As confidence grows in the method of inelastic magnetic scattering it is used to investigate increasingly complicated magnetic materials [5]. In consequence, it is important to firmly establish the exact nature of the atomic information which can be derived from a measured signal and Compton profile. To some extent, this is related to the method used to discriminate between magnetic scattering and the much more intense charge scattering.

A favoured method is to measure the contribution to the scattered signal induced by a component of circular polarization in the primary X-ray beam. This contribution is an overlap of charge and magnetic components of the amplitude for scattering. The overlap produces a larger intensity than the purely magnetic signal, it is proportional to the sign of the magnetic component of the amplitude, and it can be modulated in sign and magnitude by modulating the circular polarization in the primary beam. Regrettably, the latter is technically very demanding, at least for the moment. Less demanding, and in principle leading to the same result, is to reverse the polarity of the net magnetization of the sample by application of an external magnetic field. 
The signal in the secondary beam produced by the induced overlap of charge and magnetic amplitudes is described by a partial differential cross-section proportional to the mean helicity of the primary beam, $P_{2}$, and a linear combination of power spectra from overlap of charge and spin densities and charge and current (linear momentum) densities. Let us label electrons by the index $j$, and denote the position, linear momentum and spin operators by $\mathbf{R}, \mathbf{p}$ and $\mathbf{s}$, respectively. The charge, current and spin densities in the cross-section are,

$$
n(\mathbf{k})=\sum_{j} \exp \left(\mathrm{ik} \cdot \mathbf{R}_{\mathrm{j}}\right), \quad \mathbf{T}(\mathbf{k})=\left(-\mathrm{i} / \hbar k^{2}\right) \sum_{j}\left(\mathbf{k} \times \mathbf{p}_{\mathrm{j}}\right) \exp \left(\mathrm{ik} \cdot \mathbf{R}_{\mathrm{j}}\right)
$$

and

$$
\mathbf{S}(\mathbf{k})=\sum_{j} \mathbf{s}_{\mathrm{j}} \exp \left(\mathbf{i k} \cdot \mathbf{R}_{\mathrm{j}}\right)
$$

Note that the Hermitian conjugate $\{n(\mathbf{k})\}^{+}=n(-\mathbf{k})$, and the identity is also satisfied by $\mathbf{T}(\mathbf{k})$ and $\mathbf{S}(\mathbf{k})$ since they are built from products of commuting Hermitian operators. The operators $\mathbf{T}(\mathbf{k})$ and $\mathbf{S}(\mathbf{k})$ occur in the standard interpretation of neutron scattering by a magnetic material [6].

The power spectra in the induced overlap cross-section are formed with Hermitian linear combinations of products of $n(\mathbf{k}), \mathbf{S}(\mathbf{k})$ and $\mathbf{T}(\mathbf{k})$; it is convenient to define correlation functions,

$$
\mathbf{F}_{\mathrm{s}}(\mathbf{k}, t)=\left\langle n^{+}(\mathbf{k}) \mathbf{S}(\mathbf{k}, t)+\mathbf{S}^{+}(\mathbf{k}) n(\mathbf{k}, t)\right\rangle
$$

and

$$
\mathbf{F}_{\mathrm{m}}(\mathbf{k}, t)=\left\langle n^{+}(\mathbf{k}) \mathbf{T}(\mathbf{k}, t)+\mathbf{T}^{+}(\mathbf{k}) n(\mathbf{k}, t)\right\rangle .
$$

In these expressions angular brackets denote a thermal average value, and $n(\mathbf{k}, t)$ is the Heisenberg operator formed from $n(\mathbf{k}) \equiv n(\mathbf{k}, 0)$, and similarly for $\mathbf{S}(\mathbf{k}, t)$ and $\mathbf{T}(\mathbf{k}, t)$. Because,

$$
\{\mathbf{F}(\mathbf{k}, t)\}^{*}=\mathbf{F}(\mathbf{k},-t)
$$


the power spectrum,

$$
\mathbf{R}(\mathbf{k}, \omega)=(1 / 2 \pi \hbar) \int_{-\infty}^{\infty} \mathrm{d} t \mathbf{F}(\mathbf{k}, t) \exp (-\mathrm{i} \omega t)
$$

is purely real; the latter property is anticipated because $\mathbf{R}(\mathbf{k}, \omega)$ is a measured response function. The conjugate variable in the power spectrum, $\omega$, is the change in frequency of the $X$-rays, i.e. $\omega \neq 0$ corresponds to inelastic scattering.

The amplitude for inelastic scattering of X-rays by a magnetic material has been derived by a systematic application of perturbation theory for scattering by a bound electron. The published amplitude contains all terms up to those quadratic in electron spin and momentum operators [7]. Here, we use the cross-section derived from terms linear in these operators, which dominate at modest X-ray energies; it is a sum of various cross-sections, including those for pure charge and pure magnetic scattering proportional to power spectra of $\left\langle n^{+} n\right\rangle,\left\langle S_{\alpha}^{+} S_{\beta}\right\rangle$ and $\left\langle T_{\alpha}^{+} T_{\beta}\right\rangle$, and the induced overlap cross-section, with dimension (area/energy),

$$
\left(E^{\prime} / E\right) P_{2} r_{\mathrm{e}}^{2}\left(E / 2 m c^{2}\right)\left\{\mathbf{B}_{\mathrm{s}} \cdot \mathbf{R}_{\mathrm{s}}(\mathbf{k}, \omega)+\mathbf{B}_{\mathrm{m}} \cdot \mathbf{R}_{\mathrm{m}}(\mathbf{k}, \omega)\right\}
$$

In this expression, $r_{\mathrm{e}}$ is the classical radius of an electron, and $E=\hbar c q$ is the energy of the primary beam. The vectors $\mathbf{B}_{\mathrm{s}}$ and $\mathbf{B}_{\mathrm{m}}$ are linear combinations of unit vectors $\hat{\mathbf{q}}$ and $\hat{\mathbf{q}}^{\prime}$ $\left(\mathbf{k}=\mathbf{q}-\mathbf{q}^{\prime}\right)$ for the primary and secondary beams, weighted by the cosine of the angle $\theta$ through which the X-rays are deflected and $a=\left(1+q^{\prime} / q\right) / 2$;

$$
\mathbf{B}_{\mathrm{s}}=\hat{\mathbf{q}}^{\prime}(1-a+(\cos \theta-a) \cos \theta)+\hat{\mathbf{q}}^{\prime}(3 a \cos \theta-2 \cos \theta-a)
$$

and,

$$
\mathbf{B}_{\mathrm{m}}=-\left(k^{2} / q^{2}\right) \frac{(1+\cos \theta)}{\left(\cos \theta+q^{\prime} / q\right)}\left(\hat{\mathbf{q}}+\hat{\mathbf{q}}^{\prime} \cos \theta\right) .
$$


A derivation of (5) is found in [1,2]; an earlier discussion of its structure in the Compton limit, to which we turn later, is found in [8]. Here, we have made one small change to the presentation in $[1,2]$ by expressing the charge-current overlap contribution in terms of $\mathbf{T}(\mathbf{k})$, which is slightly more convenient to use in the present setting than the related operator chosen previously.

The invariance of the Hamiltonian that describes the electrons in the sample through the time-reversal operation, in which all velocities (including those associated with the spinning of the electrons) are reversed, gives a powerful statement about $\mathbf{F}(\mathbf{k}, t)$. Let $A(\mathbf{H})$ be an operator, which in general depends on the external magnetic field, $\mathbf{H}$, and $A^{\prime}$ the operator formed from $A$ by the operation of time reversal. The Hamiltonian $\mathscr{H}(\mathbf{H})$ satisfies $\mathscr{H}(\mathbf{H})=\mathcal{H}^{\prime}(-\mathbf{H})$. For our purposes the key identity is [9],

$$
\langle A\rangle_{\mathbf{H}}=\left\langle\left\{A^{\prime}\right\}^{+}\right\rangle_{-\mathbf{H}}
$$

By way of orientation consider its application to the total spin magnetization $\mathbf{M}=\mathbf{S}(\mathbf{k}=0)$, namely,

$$
\langle\mathbf{M}\rangle_{\mathbf{H}}=-\langle\mathbf{M}\rangle_{-\mathbf{H}}
$$

where we have used the property of $\mathbf{s}$ that it is time-odd and $\mathbf{s}^{\prime}=-\mathbf{s}^{+}=-\mathbf{s}$. If the material is paramagnetic the identity states that the field-induced magnetization vanishes as the field is reduced to zero. For a state of spontaneous magnetization $\langle\mathbf{M}\rangle$ is non-zero in the limit $\mathbf{H}=0$, and the identity states the polarity of the spontaneous magnetization is determined by the polarity of $\mathbf{H}$.

In applying (6) to correlation functions $\mathbf{F}_{\mathrm{s}}(\mathbf{k}, t)$ and $\mathbf{F}_{\mathrm{m}}(\mathbf{k}, t)$ we make use of the relations,

$$
\{n(\mathbf{k})\}^{\prime}=\{n(\mathbf{k})\}^{+}, \quad\{\mathbf{S}(\mathbf{k})\}^{\prime}=-\{\mathbf{S}(\mathbf{k})\}^{+}
$$


and,

$$
\{\mathbf{T}(\mathbf{k})\}_{H}^{\prime}=-\{\mathbf{T}(\mathbf{k})\}_{-\mathbf{H}}^{+}
$$

in which $\mathbf{p}=m \mathbf{v}(\mathbf{H})$. The important results are,

$$
\mathbf{F}_{\mathbf{s}}(\mathbf{k}, t)=\left\langle\mathbf{S}^{+}(\mathbf{k}) n(\mathbf{k}, t)\right\rangle_{\mathbf{H}}-\left\langle\mathbf{S}^{+}(-\mathbf{k}) n(-\mathbf{k}, t)\right\rangle_{-\mathbf{H}}
$$

and,

$$
\mathbf{F}_{\mathrm{m}}(\mathbf{k}, t)=\left\langle\mathbf{T}^{+}(\mathbf{k}) n(\mathbf{k}, t)\right\rangle_{\mathbf{H}}-\left\langle\mathbf{T}^{+}(-\mathbf{k}) n(-\mathbf{k}, t)\right\rangle_{-\mathbf{H}} .
$$

Hence, the correlation functions $\mathbf{F}_{\mathrm{s}}$ and $\mathbf{F}_{\mathrm{m}}$ are differences between correlation functions evaluated with opposite values of $\mathbf{k}$ and $\mathbf{H}$, and the time variable is the same in the two parts.

Spatial properties of the sample exert an influence on the dependence of its correlation functions to the sign of $\mathbf{k}$, the conjugate variable in a spatial Fourier transform. If the sample is uniform in space, which rules out microscopic samples and samples subject to inhomogeneous fields, real-space correlation functions depend on the distance between the two operators and not their individual positions. The spatial Fourier transform of such a correlation function is unchanged on replacing $\mathbf{k}$ by $-\mathbf{k}$ when the positions of the operators are also invariant through inversion, i.e. the sample possesses even space-parity and translational invariance. (E.g. sites on different sublattices of an h.c.p. structure, adopted by many elemental rare earth metals, are not interchanged by inversion). In the event that the correlation functions in $\mathbf{F}_{\mathrm{s}}$ and $\mathbf{F}_{\mathrm{m}}$ do not depend on the sign of $\mathbf{k}$ these functions change sign on reversing the polarity of the applied field. In the general case, however, to change the signs of $\mathbf{F}_{\mathrm{s}}$ and $\mathbf{F}_{\mathrm{m}}$ it is necessary to reverse both the field and $\mathbf{k}$. Under this combined operation the cross-section is unchanged, as might be anticipated, for the vectors $\mathbf{B}_{\mathrm{s}}$ and $\mathbf{B}_{\mathrm{m}}$ change sign when $\mathbf{q}$ and $\mathbf{q}^{\prime}$ are replaced by $-\mathbf{q}$ and $-\mathbf{q}^{\prime}$, respectively; rotating the target sample through $180^{\circ}$ reverses the sign of $\mathbf{k}$ relative to its axes. 
It is to be noted that the total cross-section contains other overlap terms which do not depend on the degree of circular polarization in the primary beam $[1,2]$. The terms to which we refer are anti-Hermitian linear combinations of the overlap of $n, \mathbf{S}$ and $\mathbf{T}$. The corresponding correlation functions are sums of $\mathbf{k}$ and $\mathbf{H}$ reversed correlation functions and, unlike the correlation functions in the polarization-induced overlap, do not change sign under reversal of $\mathbf{k}$ and $\mathbf{H}$.

Another representation of $\mathbf{F}_{\mathrm{s}}$ and $\mathbf{F}_{\mathrm{m}}$ can be found using the identity [9],

$$
\left\langle A_{1} A_{2}(t)\right\rangle=\left\langle A_{2} A_{1}(\mathrm{i} \hbar \beta-t)\right\rangle
$$

where $(1 / \beta)$ is the temperature in units of Boltzmann's constant. This is useful in calculating $\mathbf{F}_{\mathrm{s}}$ and $\mathbf{F}_{\mathrm{m}}$ because for each function one need calculate only a single correlation function. Also, (8) gives a physical interpretation to the effect of reversing the polarity of $\mathbf{H}$, e.g. a spin excitation reverses its helicity.

Lastly, we discuss the behaviour of the induced overlap cross-section in the Compton limit. Broadly speaking, two approaches to the task of calculating the Compton limit and corrections to it, often referred to as final state effects [10], have appeared in the literature. One approach, traditionally used in the interpretation of data on charge distributions, for which the experimental technique is well established $[11,12,13]$, relies on the calculation of specific matrix elements and a physical picture of Compton scattering in which the final state of the electron is represented by a plane wave. A second approach is to couch arguments in terms of correlation functions, and no explicit reference to a final state in the form of a plane wave. Common to both approaches is the premiss of no discernible information on spatial correlations between electrons, i.e. Compton scattering is incoherent. In the incoherent approximation to the scattering process one finds, for example,

$$
\left\langle\mathbf{T}^{+}(\mathbf{k}) n(\mathbf{k}, t)\right\rangle_{\mathbf{H}}=\left(\mathrm{i} / \hbar k^{2}\right) \sum_{j} m_{j}\left\langle\left(\mathbf{k} \times \mathbf{v}_{j}\right) I_{j}(\mathbf{k}, t)\right\rangle
$$


where the operator,

$$
I_{j}(\mathbf{k}, t)=\exp \left(-\mathrm{ik} \cdot \mathbf{R}_{j}\right) \exp \left\{\mathbf{i k} \cdot \mathbf{R}_{j}(t)\right\},
$$

arises also in $\left\langle\mathbf{S}^{+} n\right\rangle$, and $\left\langle n^{+} n\right\rangle$ which describes pure charge scattering. The two operators in (10) can be combined, using the Campbell-Baker-Hausdorff formula, to give $I_{j}$ equal to a single exponential with an argument represented as a power series in $t$. This form for $I_{j}$ is ideal for implementing the second facet of the Compton limit, namely, a very brief duration of the scattering event, in which the struck particle barely moves and its potential energy, a function of position, is unchanged to a good approximation. Calculating the argument of the exponential up to order $t^{2}$ we find,

$$
I_{j}(\mathbf{k}, t)=\exp \left\{i t\left(\mathbf{k} \cdot \mathbf{v}_{j}+\varepsilon_{j} / \hbar\right)+\frac{1}{2} \mathrm{i} t^{2} \mathbf{k} \cdot \mathbf{a}_{j}+\ldots\right\},
$$

were $\mathbf{a}$ is the acceleration operator of the struck electron.

The Compton limit of scattering is obtained from (11) by discarding terms in $t$ beyond the leading term. At this level of approximation, usually referred to as the impulse approximation [12], the current correlation function (9) and the corresponding $\mathbf{F}_{\mathrm{m}}(\mathbf{k}, t)$ share the property that $\left\{\mathbf{F}_{\mathrm{m}}(\mathbf{k}, t)\right\}^{*}$ is equal to minus $\mathbf{F}_{\mathrm{m}}(\mathbf{k},-t)$ and this leads to a purely imaginary power spectrum (response function). Since this contradicts the Hermitian character of $\mathbf{F}_{\mathrm{m}}(\mathbf{k}, t)$, which lies behind (3), we conclude that the Compton limit of $\mathbf{F}_{\mathrm{m}}(\mathbf{k}, t)$ is zero. This finding is in accord with work based on the traditional interpretation of Compton scattering found in [1] and later $[14,15]$. If we work with (11) to the order of $t^{2}$ the corresponding $\mathbf{F}_{\mathrm{m}}(\mathbf{k}, t)$ is not necessarily zero. The Compton limit of the correlation function for the overlap of charge and spin densities is the sum of,

$$
\int \mathrm{d} \mathbf{q} \exp \left\{(\mathrm{it} / \hbar)\left(\varepsilon+\hbar^{2} \mathbf{k} \cdot \mathbf{q} / m\right)\right\}\langle\mathbf{s} \delta(\mathbf{q}-m \mathbf{v} / \hbar)\rangle,
$$


evaluated for each electron. The corresponding power spectrum is the Radon transform of the spin-weighted momentum density, and its development in the language of band theory is given in [2].

Our finding that $\mathbf{F}_{\mathrm{m}}(\mathbf{k}, t)$ is zero in the Compton limit of scattering is in accord with both other theoretical developments already mentioned and empirical findings [16]. An even earlier theoretical development, using the language of correlation functions, predicted a nonzero value [17]. It can be shown that the flaw in the development lies in the representation of $\mathbf{T}(\mathbf{k})$ in terms of the orbital magnetization density, leading to incorrect symmetry properties in the Compton limit and thus a non-zero value for the overlap correlation function; in terms of Trammell's expansion [6] for $\mathbf{T}(\mathbf{k})$ the essential missing terms are those which vanish for elastic scattering.

Acknowledgements Useful discussions have been held with Dr. S. P. Collins and Dr. K. N. Trohidou and her colleagues. In the course of our work we received a draft version of [15] from the authors. 


\section{References}

[1] Lovesey S. W. 1993 Rep. Prog. Phys. 56, 257

[2] Lovesey S. W. and Collins S. P. 1996 X-ray Scattering and Absorption by Magnetic Materials (Clarendon Press, Oxford)

[3] Tanaka Y., Sakai N., Kubo Y. and Kawata H. 1993 Phys. Rev. Lett. 70, 1537

[4] Stollhoff G. 1995 Europhys. Lett. 29, 463

[5] Nakamura J., Takeda T., Asai K., Yamada N., Tanaka Y., Sakai N., Ito M., Koizumi A. and Kawata H. 1995 J. Phys. Soc. Jpn. 64, 1385

[6] Balcar E. and Lovesey S. W. 1989 Theory of Magnetic Neutron and Photon Scattering (Clarendon Press, Oxford)

[7] Grotch H., Kazes E., Bhatt G. and Owen D.A. 1983 Phys. Rev. A27, 243

[8] Platzman P. M. and Tzoar N. 1970 Phys. Rev. B2, 3556

[9] Lovesey S. W. 1986 Condensed Matter Physics: Dynamic Correlations, second edition (The Benjamin/Cummings Pub. Co. Inc., Menlo Park)

[10] Glyde H. R. 1994 Excitations in Liquid and Solid Helium (Clarendon Press, Oxford)

[11] Cooper M. J. 1985 Rep. Prog. Phys. 48, 415

[12] Kane P. P. 1992 Phys. Rep. 218, 67

[13] Sacchetti F. 1987 Phys. Rev. B36, 3147

[14] Sakai N. 1994 J. Phys. Soc. Jpn. 63, 4655

[15] Carra P., Fabrizio M., Santoro G. and Thole B. T. 1996 Phys. Rev. B53, R5994

[16] Timms D. N., Zukowski E., Cooper M. J., Laundy D., Collins S. P., Itoh F., Sakurai H., Iwazumi T., Kawata H., Ito M., Sakai N. and Tanaka Y. 1993 J. Phys. Soc. Jpn. 62, 1716

[17] Collins S. P., Cooper M. J., Lovesey S. W. and Laundy D. 1990 J. Phys: Condens. Matter 2, 6439 